\title{
Exoplanet Observations
}

Jacob L. Bean

1.1

\section{Introduction}

Observations of exoplanets have revolutionized our view of planetary system formation and evolution. Since the first unambiguous detection of an exoplanet orbiting a normal star in 1995 [1], more than 250 exoplanetary systems have been discovered. The discovery and characterization of these systems revealed that planets have a wider variety of physical and orbital properties than was anticipated. This has stimulated a critical review of every aspect of existing planet formation and evolution paradigms, and necessitated the consideration of new, more general theories.

Despite the diversity of detected exoplanets, some clear trends have emerged. In this chapter, we summarize the main results from the observational study of exoplanetary systems in the context of how they can constrain theories of planet formation and evolution. We focus on the robust findings that have emerged from examining the properties of large samples of detected exoplanets. Despite their success, current observational techniques are still quite limited, relative to what planets might be expected, and it is important to keep this in mind when interpreting results. Therefore, we also describe the limitations and biases of current observational methods and potential overinterpretations of the data that should be avoided. Compelling individual results are mentioned when they offer views beyond the well-explored parameter spaces. The rest of the chapter is separated into sections about observed exoplanet orbital, physical, and host star properties.

\section{2}

\section{Orbital Properties}

The orbital parameters of known exoplanets are perhaps the most well-determined of all their properties. The majority of the data comes from high-precision timeseries radial velocity observations, which have also yielded most of the original

Formation and Evolution of Exoplanets. Edited by Rory Barnes

Copyright (c) 2010 WILEY-VCH Verlag GmbH \& Co. KGaA, Weinheim ISBN: 978-3-527-40896-2 
discoveries. Radial velocities carry information on a planet's orbital period, eccentricity, and location and time of periastron. Information on a planet's mass is also available in radial velocity data, but this parameter is normally degenerate with the value of the planet's orbital inclination (for a notable exception, see [2]). Barring additional data from transits (see Section 1.2) or astrometry [3], what is then typically determinable is the product of the planet's mass and the sine of the orbital inclination angle ( $M \sin i)$. This corresponds to the minimum possible value for the planet's mass. When analyzing large samples of radial-velocity-detected exoplanets, the significance of the inclination angle ambiguity is reduced because of the statistical distribution of $\sin i[4,5]$. However, when considering a single planet, the uncertainty in the mass should not be ignored because there is the potential that the true mass is significantly different from the minimum or expectation values [6]. With that caveat, we drop the $\sin i$ and use $M$ when speaking about the properties of large samples of planets.

Despite being the best-known properties of exoplanets, the orbital parameters determined from radial velocity data can still potentially suffer from both large statistical and systematic errors. The published statistical uncertainties for orbital parameters are generally underestimated and rarely account for the strongly nonGaussian nature of the constraints provided by radial velocity data [7]. Systematic errors can arise when there are significant levels of stellar-activity-induced noise in the data (referred to as jitter) [8-10]. In some cases, activity signals can be periodic and coherent over typical observational timescales. Care has to be taken in the case of active stars so that not only activity-induced signals are not misinterpreted to be orbiting planets [11] but also real orbital motion signals are not incorrectly attributed to activity [12]. Systematics can also arise when all the planets in a system are not accounted for. In these cases, the determined orbital parameters for the known planets can absorb the signals from the unrecognized planets. The orbital parameters for planets later shown to be in systems harboring additional planets often undergo significant revision when the additional planets are accounted for.

Although high-precision radial velocity observations are possible around stars with spectral types from late $\mathrm{F}$ down to the bottom of the main sequence, radial velocity surveys mainly target FGK-type main sequence stars as they are the easiest to observe. High rotational speeds and activity hinder searches around stars with ages below $\sim 1$ gigayear $[13,14]$ and only a few planets are known around such stars. Therefore, the statistics of known exoplanets are dominated by those orbiting roughly solar-type main sequence stars.

1.2.1

Orbital Periods and Eccentricities

For results shown in this section, data on the orbital parameters of the currently known exoplanets (as of September 20, 2008) were taken from the Extrasolar Planets Encyclopaedia. ${ }^{1)}$ Such a composite data set is useful because it includes

1) http://exoplanet.eu/ 
data from recent discoveries and its statistics are consistent with the results from volume-limited [15] and magnitude-limited [16] surveys. We include only objects with $0.3 M_{\text {Jup }}<M<20 M_{\text {Jup }}$ and which were discovered from radial velocity surveys. The considered sample size is 204 exoplanets.

We use an upper mass cutoff for our sample above the accepted boundary between "planets" and "brown dwarfs" (the boundary for which lower mass values imply an object that cannot initiate deuterium fusion, $\sim 13 M_{\text {Jup }}$ ) because there is no clear demarcation in the mass distribution of low-mass companions to stars corresponding to this limit. What we are really interested in is in understanding the formation and evolution of objects that ostensibly formed in a circumstellar disk. As planet formation theories suggest that objects with masses greater than $13 M_{\text {Jup }}$ can form in a disk, it makes sense to consider objects out to the tail of the stellar companion mass distribution rather than an arbitrary cutoff corresponding to a theoretical internal property transition (see Chapter 7).

The lower mass cutoff of our chosen sample corresponds to the mass of Saturn. It was used to select likely gas giants and exclude low-mass planets that are part of the emerging classes of lower mass planets (i.e., Neptune-like ice giants and potentially terrestrial "Super-Earths"). Current surveys are only weakly sensitive to these types of objects compared to their higher mass counterparts, so their statistics are much more incomplete. There is some evidence that these planets' orbital properties might be different from those of gas giants, which would imply they have different formation and evolution histories as well [17-19]. The future results of ongoing planet searches will likely shed more light on this issue.

The motivation for ignoring planets discovered by transit surveys when examining the distribution of planet orbital parameters is that such searches are strongly biased toward short-period planets (i.e., $P<10$ days) because these planets are the most likely to transit in the first place, and also have multiple such events in the time spans covered by survey campaigns. Radial velocity planet searches are more sensitive to short-period planets as well, but they are still very sensitive to gas giant planets with orbital periods up to $\sim 4$ years. Therefore, radial-velocity-detected planets are the most uniform sample of planets available, although there are still biases present that should be considered (discussed further below). Disregarding the very low mass planets detected with the radial velocity method also reduces the bias toward shorter periods in the sample because their detectability is strongly dependent on their orbital period. Transiting planets do offer important information on the physical properties of planets, and these planets are discussed in Section 1.2.

The distribution of the orbital periods is shown in Figure 1.1. Inspection of this distribution reveals three potential families. One family, and the most surprising when first discovered, is planets with orbital periods of only a few days. Giant planets with such short orbital periods have come to be known as Hot Jupiters because the intensity of the stellar radiation implies that they have high equilibrium temperatures ( $>1000 \mathrm{~K})$.

The orbital parameters for short-period planets are usually very well determined due to their relatively large induced radial velocity signals and the ease of observing multiple orbits. Current thinking is that these planets did not form 


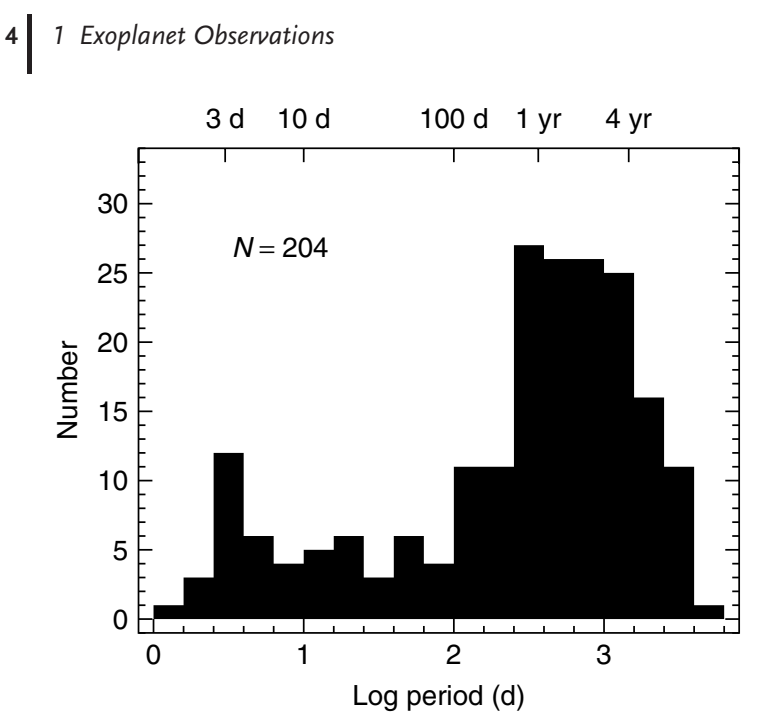

Figure 1.1 Orbital period distribution of gas giant planets identified from radial velocity surveys.

in situ, but rather they formed beyond the snow line as normal gas giants and that some subsequent evolutionary mechanism(s) brought them quite close to their host stars (see Chapter 9).

There is a well-noted "pile-up" of planets near an orbital period of 3 days. Some shorter period planets have been found, mostly from transit surveys, with the shortest known being Wasp-12 b [20] and having $P=1.09$ days. Interestingly, there appears to be a correlation between high mass planets transiting high metallicity stars with short orbital periods ( $P=1-5$ days) $[21,22]$. So it would seem that the mechanisms that gave rise to the short-period planets have a dependence on these properties, or properties that they reflect. The origin of short-period planets is one of the major issues confronting planetary evolution theory, and is discussed at length in Chapters 4-6 and 9-12.

The second observed family in the exoplanet orbital period distribution is the planets with intermediate periods ( 10 days $<P<100$ days). These planets do not display any particularly notable features, but they serve to connect the short-period planets to the longer period planets. The small, flat distribution in this range suggests that, though the orbital evolution mechanisms lead to relatively fewer planets, a continuum of results is still possible.

The third observed family is the planets with longer periods $(P>100$ days). There is a steep increase in the number of planets toward longer periods despite the corresponding decrease in sensitivity of the radial velocity method. The large, long-term surveys are only reasonably complete for gas giants with $P<4$ years, and currently have a time baseline of up to $\sim 10$ years for some objects [23]. Many systems exhibit radial velocity trends potentially due to orbiting planets with much longer periods, but the need to observe at least one full orbital cycle precludes robust characterization of most at this time [24]. 
The data on longer period planets from radial velocity surveys could be interpreted to mean that undetected gas giants with orbits similar to those of Jupiter and Saturn exist. However, precise quantification of the planet formation efficiency and probabilities of evolutionary outcomes should wait until the orbital separation parameter space is well explored. Direct imaging studies have the potential to provide complementary information to radial velocity surveys in the future, and might mitigate the problem of having to obtain decades of observations to probe for Saturn analogs.

Three planetary systems have been identified with direct imaging so far, with planets detected at separations ranging from 8 to 119 AU from their host stars [25-27]. However, these systems all orbit very young A-type stars. Currently, direct imaging results only provide constraints on massive planets having extraordinarily long periods (i.e., $P>100$ years) around young solar analogs, and even those limits depend on theoretical predictions about the spectral energy distributions of young planets that are of uncertain quality $[28,29]$. Improved technology will be needed to close the gap between radial velocity and direct imaging investigations.

In Figure 1.2, the orbital eccentricities of giant planets are plotted as a function of their orbital period, with the objects' minimum masses indicated. Regarding the eccentricity distribution, a segregation into three groups is possible, and in this case, it is guided by theory. The first group is the short-period planets. The orbits of the planets in this region all have measured $e<0.1$ (see below for some notable exceptions not included in the figure). Some of these planets even have measured $e<0.01$, which indicates that their orbits are very nearly perfectly circular. However, precise measurements (and estimations of the uncertainties

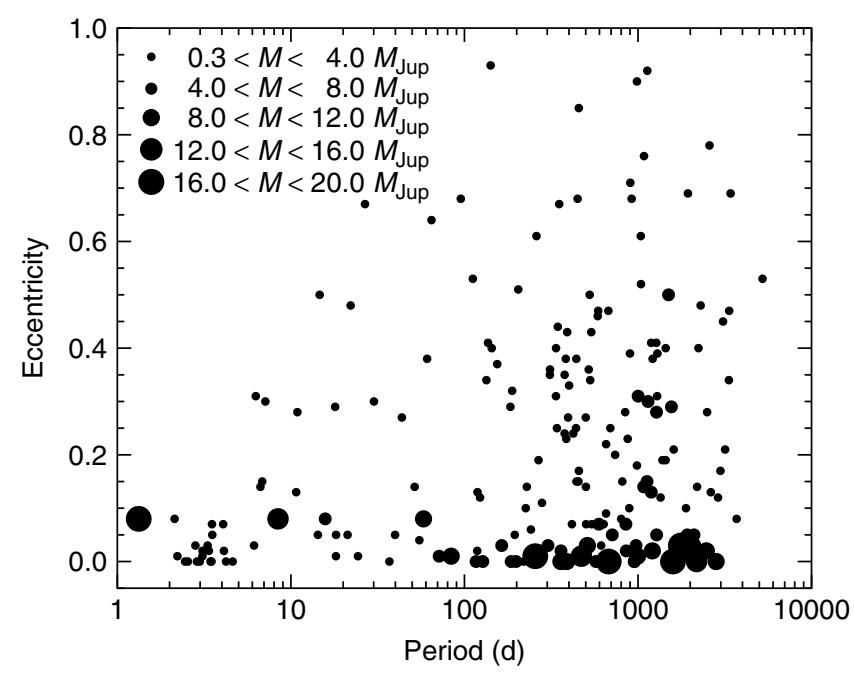

Figure 1.2 Orbital eccentricities versus period for the giant exoplanets. The symbol size indicates the minimum mass of the object. 
in these measurements) of low eccentricities are particularly difficult and require a much larger number of radial velocity observations than are usually obtained during initial discovery and characterization of planets [30]. Therefore, while the short-period planets certainly have low eccentricities, it is not known precisely to what level their orbits might be noncircular.

The second obvious eccentricity group is the long-period planets, which have eccentricities over the full range of possible values for bound orbits. The contrast between the eccentricities of the short-period planets and those of the longer period planets suggests that a mechanism operates to affect one distribution and not the other. This mechanism is widely believed to be forces arising from tidal interactions between the planets and their host stars. These forces act on the short-period planets to circularize their orbits on timescales shorter than the age of the system. On the basis of this theory, there is likely a third eccentricity group composed of planets in the transition region between the fully circularized planets and those not noticeably affected by tidal forces. The orbital eccentricities of these planets are damped somewhat, and therefore they have a smaller range of eccentricities than the longer period planets, but not enough time has elapsed for their orbits to become completely circularized. The results of a thorough study of the period-eccentricity relationship for exoplanets using data of fairly uniform quality indicate that the circularization cutoff occurs around $P=5$ days, the transition region spans 5 days $<P<100$ days, and nontidally affected planets have $P>100$ days [31].

There are some notable outliers from the nearly circularized orbits of shortperiod planets trend, all of which were excluded from the data set used for the plots in this section. A number of hypothesis have been proposed to explain the origins of these planets' eccentricities including eccentricity pumping from regular dynamical interactions with another planet or a stellar companion, and longer tidal circularization timescales than the age of the systems. More details on the theory of tides and the interpretations of the observed eccentricities of short planets can be found in Chapter 12.

Another general property of giant planets discernible from Figure 1.2 is the trend for the most massive planets to rarely be in short-period orbits. In the data considered here, only two objects have $M>5 M_{\text {Jup }}$ and $P<50$, and both orbit one component of a multistar system (see Section 1.2.3). A few other similar planets have been found from transiting planet searches, but the trend seen here is still statistically significant when those objects are included. The rarity of massive, short-period planets could be a clue about the orbital evolution mechanism that moves gas giants inward from their birth environment.

\subsection{2}

\section{Multiplanet Systems}

The orbital parameters of planets in multiplanet systems are especially important constraints for theories of planet formation and evolution (see Chapters 4-6). However, detection and characterization of such systems are more challenging. As 
mentioned above, signals from additional planets in a system can often be hidden because they are absorbed by the orbital parameters of the known planets when modeling radial velocity data. Generally, for each additional planet in a system, roughly twice as much radial velocity data are needed to constrain the planets' orbits. The amount of needed data increases as the signals from the additional planets decrease, and detection of additional low-mass planets in known planetary systems is particularly challenging.

In some cases, two or more planets in a multiplanet system can be gravitationally interacting. This leads to non-Keplerian motion like periastra precession and eccentricity variations on a wide range of timescales. Most such systems only exhibit interactions on long timescales (i.e., much longer than an observational timescale, see Chapter 3). For these systems, an observationally determined model provides a snapshot of the system and direct integration of the equations of motion is needed to study secular behavior [32]. The implied stability of the determined orbital parameters can also be an issue, with the "best-fit" values sometimes describing an unstable system [33-35]. As nearly all exoplanet systems have existed for at least 1 gigayear, such orbital parameters are assumed to be spurious. Stability considerations are sometimes used in these cases to help identify a more likely model. However, such a process involves weighing stability against observational data and there is no straightforward method to incorporate stability in a robust statistical manner (for one such method see [36]). Therefore, modifications to orbital parameters based on stability criteria are sometimes based on judgment and their uncertainties are difficult to quantify.

Multiplanet systems that exhibit planet-planet interactions on short timescales (i.e., similar to or shorter than observational timescales) are rare, but they do exist. For these systems, dynamical models based on the direct integration of the equations of motion must replace the normal Keplerian models when fitting observational data $[37,38]$. The proposed existence of an additional very low mass planet $\left(\sim 5 M_{\oplus}\right)$ in a known planetary system that was later refuted is an extreme example of how erroneous results can be obtained when neglecting the signature of planet-planet interactions [39-41]. A more subtle pitfall is that the orbital parameters determined for these multiplanet systems using a Keplerian model can be inappropriate for studies assessing their long-term dynamics and stability zones.

Despite the challenges of using dynamical models to fit observational data, the systems requiring such analyses present an opportunity to obtain more information from typical observations. If the dynamical perturbations are large enough, and occurring on a short enough timescale, then study of radial velocity data can yield constraints on the interacting planets' true masses and degree of coplanarity $[37,38,42]$. The key to the additional insight is that the planet-planet perturbations discernible in radial velocity data are dependent on the true masses and three-dimensional orbital orientations of the interacting bodies. Therefore, if the perturbations can be characterized well enough, then the planet masses and orbits can be constrained. To date, this kind of analysis has only been applied to one 
system (GJ 876, [2, 42]), but this should also be possible for some other known systems when enough data have been collected.

In all the cases of multiplanet systems, the unknown orbital orientations are a problem. The true masses of planets are needed to accurately calculate the dynamical characteristics of multiplanet systems. However, these are rarely known due to the orbital inclination angle degeneracy with planet mass in radial velocity data. Another issue is that the degree of coplanarity of the orbits of planets in all multiplanet systems (except the one mentioned above) is not known. The unknown orbital plane alignments affect the dynamical characteristics of a system. As they are without observational constraint, studies of the dynamics of multiplanet systems have to assume planet masses (usually the minimum or expected values) and the orbital orientations (usually perfectly coplanar).

Currently, there are 33 well-characterized multiplanet systems and $\sim 30 \%$ of systems with only a single identified planet show significant evidence for multiplicity [43]. This percentage must be considered a lower limit to the frequency of multiplanet systems because of the incompleteness of surveys for lower mass and longer period planets. One system is known with five planets (55 Cnc [44]), one is known to harbor four ( $\mu$ Arae [35]), and the others harbor two or three. The orbital eccentricities in systems known to have three or more planets (median $e=0.13$ ) are typically lower than those of planets in other systems (median $e=0.25)[43,45]$. This suggests that dynamical instabilities (see Chapter 11) could play an important role in the evolution of planetary systems because the higher order multiples that survive are intrinsically more stable.

A significant number ( 25\%) of the multiplanet systems have two planets in or near mean motion resonances [46]. The most common is $2: 1$, with $3: 1,4: 1$, $5: 1$, and $3: 2$ also represented. The current best explanation for the observed resonances is differential migration in a disk leading to mutual capture ([47], and see Chapter 9). The prevalence of resonant configurations suggests that this is a common outcome of planetary formation and evolution, and that the responsible mechanism must arise naturally from normal conditions.

1.2 .3

Planets in Multiple Star Systems

A significant fraction of stars in the Galaxy are part of a multiple star system [48, 49], and a close stellar companion could disrupt planet formation or influence planets' orbital parameters. Therefore, a complete picture of planet formation and evolution must include consideration of planets around stars that have additional stellar companions, and the details of the effects of stellar multiplicity should be constrained by observations.

Currently, more than 30 exoplanet host stars are known to have stellar companions $[50,51]$. Most of the known stellar companions are either widely separated stars of various types, or close-in stars with low masses [52]. The predominance of low-mass stars among close-in companions is likely at least partly an observational bias because radial velocity planet searches mostly exclude near-equal mass binary 
stars with separations $<5^{\prime \prime}$. The main reason for this is that for all binaries but those with a high mass ratio (large brightness contrast), the obtained spectra contain significant contributions from both objects. These spectra are confusing for the normal algorithms used for high-precision radial velocity measurements. Therefore, the close-in companions to exoplanet host stars have to be sufficiently dim so that they do not affect normal high-precision radial velocity measurements. Many of these close-in stellar companions were not even known a priori, but were discovered with a targeted search after an exoplanet had been found [53].

Some techniques have been developed to overcome the problem of blended spectra from close multiples where the components have similar masses [54, 55], but these have seen limited application. On the basis of these methods [56], a planet was claimed to have been detected around the primary of a close triple star system (HD 188753A), but this is controversial because with the other [57] method the planet was not detected. This further illustrates the difficulty of reliably detecting planets around close multiples.

Among the noncontroversial exoplanet detections, three orbit host stars with a stellar companion separated by 20 AU (G) 86 b [58], $\gamma$ Cep b [59], and HD 41004 b [60]). These systems pose challenges for planet formation theories because such a close companion could truncate the star's circumstellar disk such that gas giant formation via core accretion becomes unlikely [61, 62].

The orbital properties of exoplanets in multistar systems do exhibit some differences from planets orbiting ostensibly single stars. The main difference is that most of the massive $\left(M>2 M_{\text {Jup }}\right)$ short-period ( $\mathrm{P}<40$ days) planets orbit a star in a multistar system (see Figure 1.2) [52, 63, 64]. Additionally, all short-period planets in multistar systems are in nearly circular orbits $(e<0.05)$ [52, 64]. There is also the suggestion that intermediate-mass planets $\left(2<M<6 M_{\text {Jup }}\right)$ are more common in multistar systems [52]. No correlation has been noticed for planet and companion star orbital properties.

The statistics of planets in multistar systems are still limited owing mainly to the difficulty of detecting planets in close binary systems. Therefore, the observed trends and lack of trends must be treated as preliminary. Continued searches for planets in multiple systems, as well as searches for stellar companions to exoplanet host stars, are needed. In particular, the question of low-mass planets in multistar systems is still very open.

1.2.4

\section{Spin-Orbit Alignment}

Radial velocity data for transiting exoplanets offer an additional constraint on their orbital configuration beyond the usual parameters discussed above. The additional information is the angle $(\lambda)$ between the planets' orbital axes and the rotational axes of their host stars, or their "spin-orbit" alignment. This angle is measurable due to the subtle affect of an eclipse of a small portion of a star's surface on its spectrum. 
As a planet transits a rotating star, parts of the star with different line-of-sight velocities are obscured. These obscurations lead to changes in spectral line profiles because some component of the Doppler broadened line is missing. Radial velocities measured during a transit then display spurious shifts due to the line profile distortions. If a planet's orbit is prograde, then as it transits its host star, the stellar radial velocities will show a red shift during the first half as some of the blue-shifted light is missing, and then during the second half of the transit the stellar radial velocities will show a blue shift as some of the red-shifted light is missing. The signature of this phenomenon in radial velocities is known as the Rossiter-McLaughlin (RM) effect $[65,66]$. The morphology of the RM effect depends on the geometry of the orbit crossing and the rotation of the star [67]. The size of the effect is of the order of tens of meters per second for a Hot Jupiter transiting a main sequence G-type star. Therefore, the effect can be characterized for many transiting planets with current technology, and this characterization yields the spin-orbit angle.

Table 1.1 summarizes the measurements of the RM effect for transiting exoplanets. The main result is that all but one of the exoplanets orbit in the prograde direction and have a spin-orbit angle that is close to or consistent with $0^{\circ}$. These findings are an important constraint for theories of planet formation and evolution because they suggest that stars and their planets originate and inherit their angular momentum from the same collapsing molecular cloud, and that subsequent evolution does not significantly disturb the planets' orbits from their original plane. Of the planets orbiting in the prograde direction with small spin-orbit angles, only HD $209458 \mathrm{~b}$ has a more than $3 \sigma$ detection of a nonzero angle $\left(\lambda=-4.4^{\circ}\right.$ $\left.\pm 1.4^{\circ}\right)$. This value is of the same scale as the spin-orbit angles for the planets of the solar system, which implies some commonalities despite other very different characteristics.

The one possible exception to the spin-orbit alignment rule is $\mathrm{XO}-3 \mathrm{~b}\left(\lambda=37.3^{\circ}\right.$ $\pm 3.7^{\circ}$ ). This planet is also unusual for its definite nonzero eccentricity despite its short-period orbit (see Section 1.2.1), and it is also one of the few massive close-in planets $\left(M=12.5 \pm 1.9 M_{\text {Jup }}, P=3.2\right.$ days [77]). It could be that this object is part

Table 1.1 Measurements of the spin-orbit angle for transiting exoplanets.

\begin{tabular}{lrl}
\hline Planet & \multicolumn{1}{c}{$\lambda\left(^{\circ}\right)$} & Source \\
\hline HD 209458 b & $-4.4 \pm 1.4$ & {$[68]$} \\
HD 189733 b & $-1.4 \pm 1.1$ & {$[69]$} \\
HD 147506 b & $0 \pm 12$ & {$[70,71]$} \\
TrES-1 b & $30 \pm 21$ & {$[72]$} \\
HD 149026 b & $-12 \pm 15$ & {$[73]$} \\
CoRoT-2 b & $7.2 \pm 4.5$ & {$[74]$} \\
HD 17156 b & $9.4 \pm 9.3$ & {$[75,76]$} \\
XO-3 b & $37.3 \pm 3.7$ & {$[77,78]$} \\
TrES-2 b & $-9 \pm 12$ & {$[79]$} \\
HAT-P-1 b & $3.7 \pm 2.1$ & {$[80]$} \\
& & \\
\hline
\end{tabular}


of a rare class of planets that had different formation or evolutionary experiences than most of the other detected exoplanets, but this is only speculation. Such counterexamples to otherwise robust statistical trends illustrate the challenges of improving our understanding of the complex interplay of different physical effects leading to planet formation and evolution by studying the inhomogeneous and incomplete sample of known exoplanets.

1.3

\section{Physical Properties}

The main observable physical properties of exoplanets are their masses, and, in some cases, their radii. These data constrain planet formation mechanisms, which are generally expected to govern the resulting masses and structures of planets. However, in some individual cases, evolutionary processes could have also played a critical role and must be kept in mind when interpreting results.

\subsection{1}

\section{Mass Distribution}

As described above, the minimum masses of exoplanets can be determined from radial velocity data, and this information is sufficient for statistical studies of large samples of exoplanets. We have already discussed some aspects of how the orbital properties of gas giants depend on planet mass, and now we turn to examining the overall mass distribution.

Figure 1.3 is a plot of the mass function for all known radial-velocity-detected exoplanets, with the data again taken from the the Extrasolar Planets Encyclopaedia. The dominant population is planets with masses on the order of Jupiter's mass. These planets are expected to be composed predominantly of gas, which is confirmed by mass and radius measurements for the subsample of transiting planets in this mass range (Section 1.2.2). The median of the sample is $1.7 M_{\text {Jup }}$, and planets with masses up to $10 M_{\text {Jup }}$ are not uncommon.

A simple interpretation of the gas giant mass distribution suggests that massive planet formation is generally more efficient than it was in the solar system. However, it is important to consider the biases of radial velocity planet searches to higher mass and shorter period planets when interpreting the statistics. With current radial velocity precisions, the large surveys cannot easily detect Saturn-mass planets $\left(\approx 0.3 M_{\text {Jup }}\right)$ with orbital periods longer than about 300 days [81]. So some fraction of lower mass gas giants might be missing even in the orbital parameter space nominally covered by radial velocity surveys.

Regarding the issue of the short-period bias, nearly all the gas giant planets known likely moved significant distances from their original formation location. If this disturbance was due to migration arising from friction with a disk (see Chapter 9), then the massive gas giant planets might have experienced gas accretion much longer than planets that did not migrate large distances. So the mass distribution 


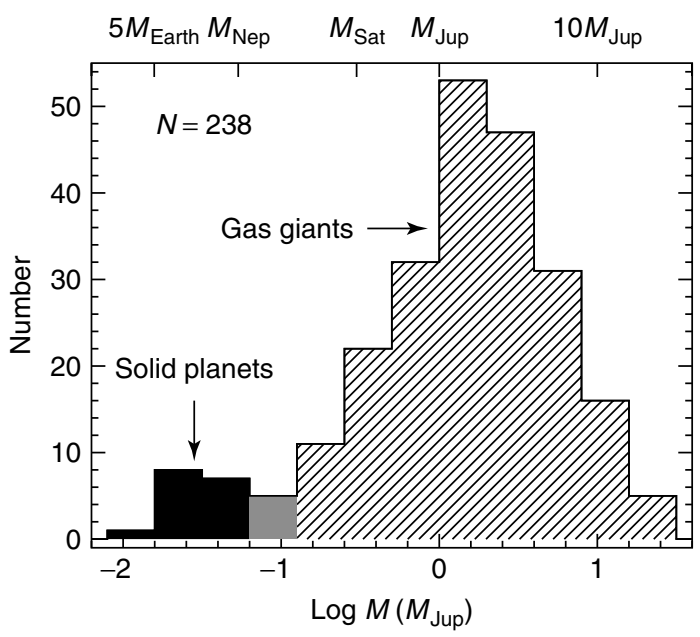

Figure 1.3 Mass distribution of all known exoplanets. There is likely a boundary separating mostly gaseous planets from mostly solid planets around $30 M_{\oplus}\left(\approx 0.1 M_{\text {Jup }}\right)$, although its exact location is uncertain.

of the known gas giant planets might be different from the mass distribution of the longer period ones yet to be discovered. Despite the biases, the large numbers of detected gas giants imply that their formation is not unusual and must arise from fairly high likelihood events.

Recently, radial velocity surveys have begun to detect lower mass planets that are likely a separate population from the gas giant planets. These planets are expected to be composed mostly of heavy elements (ices and rock). Therefore, they might be referred to as solid planets. The likely solid compositions for objects in this mass range are reinforced by the $\sim 90 \%$ heavy element abundance inferred for the one known transiting planet in the sample, the $23 M_{\oplus}$ GJ $436 \mathrm{~b}$ [82].

The exact boundary between the gaseous and solid planets is uncertain, but inspection of the exoplanet mass distribution reveals a potentially significant gap around $30 M_{\oplus}\left(\approx 0.1 M_{\text {Jup }}\right)$. As these two groups of planets are thought to have very different formation scenarios, it is possible that they differ in other observable properties. Although the sample of solid planets is severely incomplete, even at the shortest periods, there is a hint that the metallicities of the stars hosting these planets are different from those hosting gas giants [83] (see Section 1.3.1 for more discussion). It will be interesting to compare the orbital parameters of the two samples as more solid planets are detected and characterized. In the future, it might become possible to distinguish the mass distributions of rocky "Super-Earth" planets from icy Neptune-like planets.

Interestingly, two out of the seven planet detections from microlensing observations have been of very low mass planets $\left(3\right.$ and $\left.5 M_{\oplus}\right)[84,85]$. These findings suggest that such planets might be quite common because microlensing events are so rare that a single detection points to a large underlying sample. Therefore, 
the low-mass planets are similar to the long-period planets in that they represent a potentially large sample of still-to-be-discovered planets. The possible extensive incompleteness of the known planet population should be kept in mind when constraining theory. Simulations of planet system formation and evolution tuned to reproduce the orbital and physical properties of currently known exoplanets might be missing the bigger picture by focusing on what turns out to be the minority of planet populations.

\section{3 .2}

\section{Masses and Radii of Transiting Planets}

If a planet is observed to transit its host star, then photometric time-series observations of an event (i.e., a transit light curve) can be used to determine the planet's radius and orbital inclination. This knowledge yields the true mass and density of the planet when combined with the minimum mass obtained from radial velocity data. The mass and radius of a planet is a very interesting data set because it allows inference of the planet's internal structure and composition. Transiting planets also afford a view into their atmospheric properties via timeresolved photometry and spectroscopy as they pass in front of and behind their host stars. These data mainly concern the planets' atmospheric chemistry and are discussed in Chapter 8.

Figure 1.4 is a plot of the masses and radii of all the known transiting objects with $M<20 M_{\text {Jup }}$. The data were taken from a compilation [22] and recent individual results $[20,86-103]$. The most striking thing about the mass-radii data is that they reveal that Jupiter-mass planets can have a wide range of densities - up to an order of magnitude in dispersion. All of these planets orbit very close to their host stars, so the effect of significant stellar insolation must be taken into account when modeling them. Nevertheless, current models suggest that the range of densities observed is more due to natural variations arising from the planets' different heavy element abundances rather than the different levels of insolation they receive [104]. The heavy elements are thought to be primarily concentrated in a central core, and so planets of the same mass and different densities are expected to have different-sized solid cores (see Chapter 8).

Figure 1.5 shows the estimated core masses for the transiting Jupiter-mass planets from a homogeneous study of transiting planet properties [22]. It should be noted that these values are heavily dependent on theoretical models of uncertain accuracy, and that when different sets of models are used different results may be obtained. However, the diversity of radii seen for these planets definitely implies core masses over a large range. In the case shown, the estimated values range from 0 to $70+M_{\oplus}$.

The inference of nonzero core masses and heavy element enhancements of many planets has mixed interpretation. Significant cores are a prerequisite of gas giant formation through the core accretion mechanism (see Chapter 5), so the data seem to favor that model. However, simulations of gas giant formation via disk 


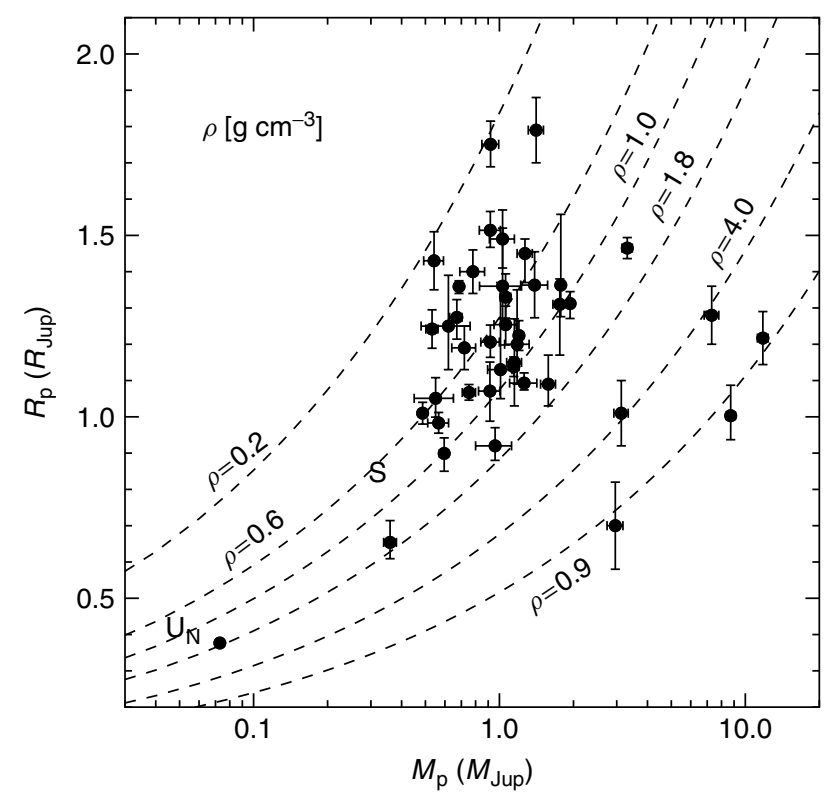

Figure 1.4 Mass-radius diagram for all known transiting exoplanets. The dotted lines are contours of constant density. The locations of Jupiter, Saturn, Neptune, and Uranus are indicated by the labels "J", "S", "N", and " $U$ ", respectively.

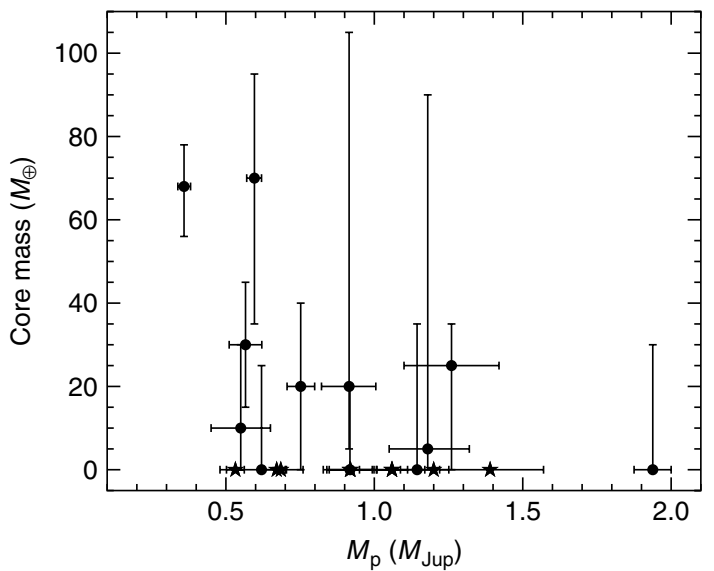

Figure 1.5 Estimated core masses for some transiting exoplanets (data from [22]). The circles represent planets with nonzero or potentially nonzero core masses. The stars represent planets with radii larger than those suggested by coreless models. 
instability (see Chapter 4) suggest that sedimentation of solids and planetesimal accretion could potentially lead to the formation of cores in these planets.

A particularly important issue in this area is that the radii of some of the lowest density planets cannot be reproduced even by coreless models - these planets are inexplicably large. The first discovered transiting planet, HD 209458 b [105], has become the prototype of these so-called inflated Hot Jupiters. Its radius is $0.2 R_{\text {Jup }}$ larger than nominal coreless models that include insolation [104]. A number of other transiting planets that follow the same trend are now known. So the effect likely arises from a general property of these planets, rather than each of them being unique exceptions. A leading explanation for the larger than expected planets is that they have an unaccounted for source of internal heat [106]. One source could be energy dissipated from ongoing or previous orbital circularization by tidal interactions [107, 108] (see Chapter 12 for more discussion). However, the question of why some Hot Jupiters are inflated is still a major outstanding issue as no consensus on a single mechanism has been reached. A related point is that if coreless models could reproduce some of the planets' sizes, then that would be evidence that they formed via disk instability.

The high end of the distribution of transiting planet densities also offers surprising information. An interesting example is the planet HD 149026 b [109] because heavy elements probably constitute roughly half of its entire mass [110]. Such a high abundance of heavy elements in HD $149026 \mathrm{~b}$ and other planets is potentially problematic even for the core accretion mechanism [111, 112], although not all authors agree with this assessment [113]. It has been suggested that the planets with enormous cores could be the products of collisions between planets. This evolutionary scenario suggests the existence of outer planets in the systems. Future observations will be able to test the collision origin theory by looking for these purported companions.

The search for correlations among the properties of transiting planets is still in its preliminary stages. All the investigations to date have utilized small samples of planets, many of which have nonnegligible uncertainties in their measured physical properties, and also theoretical models that are of uncertain quality. As more transiting planets are found and models are improved it will be important to reevaluate the results in this area. The question of the structure of transiting planets is taken up again in Chapters 8 and 12. See also Section 1.3.1 for a discussion of exoplanet host star properties in relation to planet statistics.

\section{4}

\section{Host Star Properties}

Stars and their planets are thought to arise from the same fragment of a collapsed molecular cloud. Consequently, a star's properties likely reflect the physical conditions of its circumstellar disk. The study of correlations between planets' and their host stars' characteristics can therefore give an insight into the 
planet formation and evolutionary processes. In this section, we consider results on the correlations between planets and their host stars' compositions and masses.

1.4.1

Planet-Stellar Composition Correlation

The investigation of planet host stars' atmospheric chemical compositions has yielded important constraints on planet formation theories. The most significant finding in this area has been the observed strong correlation between stars' iron abundances $^{2)}$ and the frequency of giant planets detected with the radial velocity method [114-116]. Iron is an important element because it traces a star's overall metal content (or metallicity) reasonably well and exhibits numerous lines in the optical spectra of solar-type stars that can be analyzed for determination of abundance. Most studies in this area use a star's iron abundance as a proxy for its metallicity and suggest that the giant planet frequency is correlated with overall metal content rather than iron specifically. This is not strictly correct as the determination of true metallicity requires at least an assessment of the abundances of all the elements that are typically more abundant than iron in stellar photospheres (C, N, O, Ne, Mg, and Si). Such detailed analyses have been carried out for some samples of planet-hosting stars and a few small compositional anomalies have been found (one of which is described below), but mainly the results have confirmed the overall metallicity correlation with iron abundance typically found in field stars $[117,118]$. Therefore, the iron abundance trend seen in planet-hosting stars is appropriately interpreted to be broadly a trend with overall metal content.

Figure 1.6 shows data on the giant planet detection frequency as a function of iron abundance for FGK-type dwarf stars from one of the large planet search programs [116]. The sample is limited to FGK-type dwarf stars because these are the stars that make up the majority of planet search targets. Therefore, these are the stars for which the best statistics can be obtained. These stars also have similar masses, so the sought-after correlation between composition and planet frequency is not polluted by another possible correlation with that parameter (see Section 1.3.2). Furthermore, the spectra of FGK-type dwarfs are similar enough so that the same type of abundance analysis method can be used on them. The focus is on giant planets in this correlation study because these are the planets for which robust detection/nondetection statistics are available, and also because they should have a similar formation and evolutionary history.

Numerous studies have reached the conclusion that giant planets are detected more often around metal-rich stars $[115,119,120]$. The origin of the trend has

2) Stellar photospheric chemical abundances are typically given as the logarithm of the fractional abundance relative to the hydrogen abundance and on a scale relative to the solar value.
The notation is $[\mathrm{X} / \mathrm{H}] \equiv \log _{10}\left(N_{\mathrm{X}} / N_{\mathrm{H}}\right)_{\star}-$ $\log _{10}\left(N_{\mathrm{X}} / N_{\mathrm{H}}\right)_{\odot}$, where $N_{\mathrm{X}}$ is the number density of element $\mathrm{X}$ and $N_{\mathrm{H}}$ is the number density of hydrogen. 


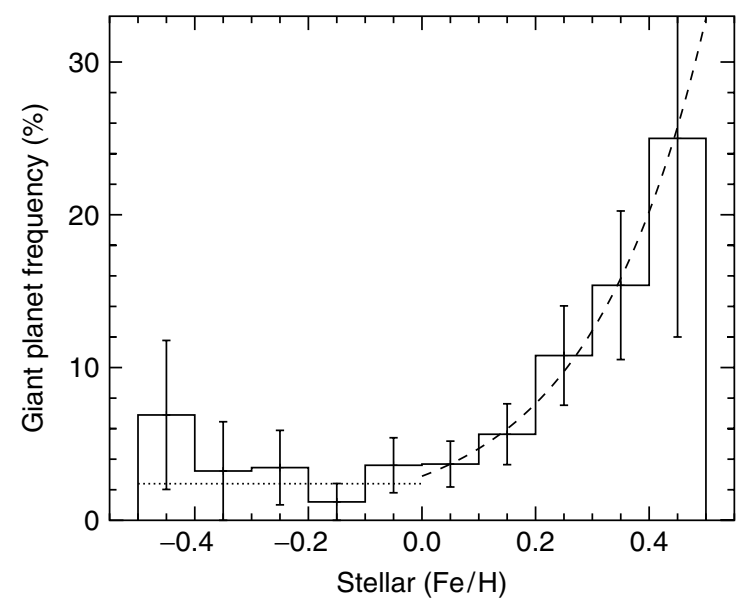

Figure 1.6 The frequency of giant planet detection as a function of stellar iron abundance (data taken from [116]) with errors based on Poisson statistics. The dotted line is a constant fit (2.4) to the data for $[\mathrm{Fe} / \mathrm{H}]<0.0$, and the dashed line is a power law fit $\left(2.9 \times 10^{2.1 \times[F e / H]}\right)$ for $[\mathrm{Fe} / \mathrm{H}]>0.0$.

been the subject of much debate, and there have been three main competing explanations. The first idea, which is known as the primordial hypothesis, is that the metallicities of the stars reflect the original composition of the protostellar cloud. In this case, giant planets would seem to form preferentially out of circumstellar disks with higher heavy element content. The second idea, which is known as the pollution hypothesis, suggests that the formation and/or migration of giant planets leads to postformation accretion of gas-depleted material onto the host star. The third idea suggests that the observed trend is a selection effect arising from planets being easier to detect around metal-rich stars because obtainable radial velocity precisions are better for stars with more and stronger lines in their spectra.

Most of the current data firmly show that the primordial hypothesis is the correct one. The supporting results include the lack of a metallicity trend with outer convective zone size among main sequence stars or along the subgiant branch [116, 121]; no observed trend of individual elemental abundances with different condensation temperatures [116, 122]; no degradation of radial velocity precision over the metallicity range covered by radial velocity planet searches $[116,121]$; no metallicity trend with planet orbital period [116]; no differences in the abundances between binary stars or stars in a cluster [123-125]; no evidence of ${ }^{6} \mathrm{Li}$ in the photospheres of planet-hosting stars [126]; and asteroseismologic inference that a planet-hosting star's high metallicity is not just concentrated in its atmosphere [127].

It has been pointed out that a single analytic function cannot provide a good fit to the giant planet frequency as a function of host star iron abundance [128]. Instead, a two-piece function with a constant frequency in the subsolar abundance region and a power law for the supersolar region is indicated (see Figure 1.6). This 
suggests a dichotomy in giant planet formation. For stars with metal abundances equal to or less than the solar value, the frequency of giant planet formation is $\sim 2 \%$ and is independent of metal abundance. For stars with more metals than the Sun, the giant planet formation rate has a steep correlation with metal content and is $\sim 25 \%$ for stars with three times the solar metal abundance.

The discovered giant planet-metallicity correlation and the evidence for the primordial explanation is an important constraint for planet formation theories. However, like the possible presence of heavy element cores in transiting planets, the appropriate interpretation of the data is still uncertain. The data could be interpreted as evidence that core accretion rather than disk instability is the dominant mechanism for the formation of the known gas giants because of the assumption that higher stellar metallicity implies circumstellar disks with a higher surface density of solids. The rocky cores that are the seeds of giant planets in the core accretion model grow faster in such environments. This means the cores can reach the critical size necessary to initiate runaway gas accretion faster. As giant planet formation is a race against the disk gas dissipation, the faster the cores can grow the more giant planets will form [129]. On the other hand, the expected correlation of giant planets with stellar metallicity in the disk instability mechanism is more uncertain, with different authors suggesting that there should [130] or should not [131] be a correlation. See Chapters 4 and 5 for more detailed discussions of the two different gas giant formation mechanisms, and the possible interpretation of the metallicity distribution of exoplanet host stars.

Under the core accretion paradigm, there is most likely a cutoff for the surface density of solids below which giant planets will not form around a solar-type star. This implies the existence of a lower limit for the metallicities of giant planet hosts [132]. Currently, there are three giant planet hosts with [Fe/H] 0.7 (HD 114762 [133], HD 47536 [134], and HD 155358 [135]). However, the low-metallicity regime of planet hosting stars has not been well studied, mainly because of selection effects $[133,136]$. Therefore, the three lowest metallicity stars might not correspond to the lower limit for giant planet formation.

Another interesting result in this area is that the heavy element abundances of transiting planets and their host stars seem to be correlated [22, 137, 138]. However, what is seen is not a simple linear style relationship, but an increase in the upper limit of the core mass distribution toward higher host star metallicities. This observed trend also suffers from many of the same problems as the other properties of transiting planets mentioned above, and so will need to be reevaluated as more and better data become available.

With regard to individual element enhancement for planet-hosting stars, there has been a finding that giant planet host stars have higher silicon and nickel abundances for a given iron abundance than field stars [139]. Following the primordial argument, it would seem that these enhancements further increase the giant planet formation efficiency. However, some caution is warranted in relying too heavily on this result because it is from a single study and its significance, while high, is not overwhelming. 
The recent emergence of a sample of potentially solid exoplanets has also motivated study of the compositions of their host stars. There is a preliminary result that such planets are not found preferentially around metal-rich stars, which is in contrast to the trend for giant planet host stars [83]. The current result relies on a small sample of detected planets and therefore suffers from small number statistics. There is also the problem that half of all detected solid planets orbit $\mathrm{M}$ dwarfs and this brings up the question of the planet formation correlation with host star mass (see Section 1.3.2). Nevertheless, the composition of solid planet hosts is likely to be a very important area of research as more of these planets are discovered. See Chapter 6 for a detailed discussion of terrestrial planet formation theories.

\section{4 .2}

\section{Planet-Stellar Mass Correlation}

It is possible that circumstellar disk mass is proportional to stellar mass. If so, then host star masses are another property that might be correlated with planet properties because total disk mass is likely an important parameter governing planet formation. However, searching for a trend in this area is not straightforward due to the challenges of detecting planets around stars with a wide range of masses. As mentioned above, radial velocity surveys target mostly late F- to mid K-type stars, but these stars only cover a relatively small range of masses. Radial velocity surveys aimed at $\mathrm{M}$ dwarfs [140-142], and subgiants and giants [17, 143-145] probe a wider range of masses, but include fewer stars and have different sensitivities and selection biases than the FGK-type star surveys. Nevertheless, there are preliminary data that suggest that the giant planet detection frequency is correlated with stellar mass.

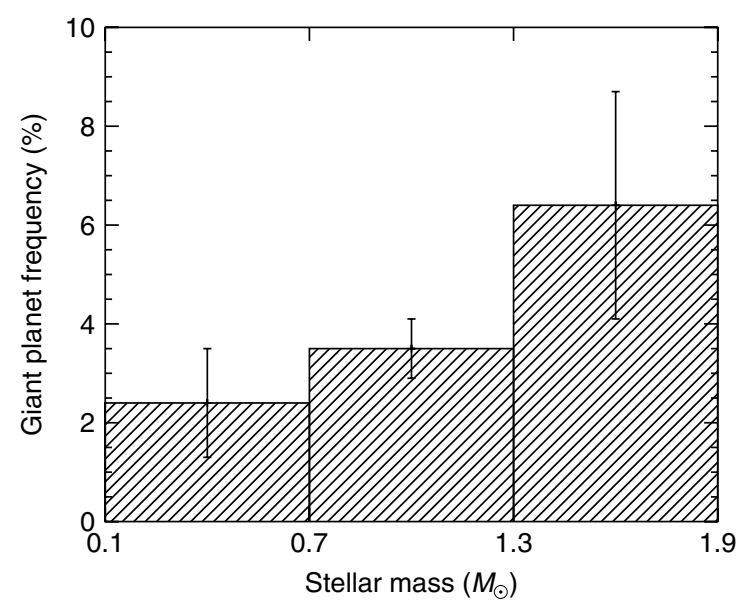

Figure 1.7 The metallicity-corrected frequency of giant planet detection as a function of stellar mass with errors based on Poisson statistics (data taken from [17]). 
Figure 1.7 shows the frequency of giant planet detection as a function of stellar mass in one of the large planet search programs (data taken from [17]). There are two caveats to these statistics. First, the data are corrected for stellar metallicity based on the observed trend for solar-type stars. This seems appropriate and necessary, but there is the problem that stellar abundance analyses potentially suffer from systematic errors when applied to different areas of stellar property parameter space [146]. Photospheric abundances for low-mass stars are particularly troublesome to obtain on a scale consistent with solar-type stars because their optical spectra are dominated by molecular lines [147]. Given the steep dependence of planet detection frequency on metallicity in the supersolar regime (see Section 1.3.1), small systematic errors in the metallicities for the mass-correlation sample could be magnified in the applied corrections for these data.

Another caveat is that the detection limits for planets orbiting the high-mass stars are not as stringent as for planets around the solar-type and lower mass stars because the radial velocity signature for a planet of a given mass is inversely proportional to the host star mass. Also, surveys targeting high-mass stars only have about half the time baseline as $M$ dwarf surveys, and about a third of the time baseline as FGK-type star surveys. Therefore, it has been recommended to treat the lower error range in the high-mass bin for the data in Figure 1.7 as a lower limit to the planet detection frequency in this range [17].

From the data in Figure 1.7 it appears that giant planets are more often found around massive stars. Taking the data at face value, giant planets are nearly three times more frequent around stars twice as massive as the Sun than stars with half the mass of the Sun. This is consistent with a number of other findings [140-142, 145], and no evidence to the contrary exists.

Perhaps even more telling than the above data is the fact that there have been no Hot Jupiter detections for M dwarfs despite the ease with which these planets could be detected, given the large radial velocity signal they would have. Assuming $300 \mathrm{M}$ dwarfs have been surveyed for Hot Jupiters [17], no detection implies a $1 \sigma$ upper limit to their frequency of $0.4 \%$. In contrast, the frequency of Hot Jupiters around FGK-type stars is $1.2 \% \pm 0.2 \%[16]$.

As it stands, the observed giant planet frequency correlation with stellar mass is potentially in agreement with the core accretion model and inconsistent with the disk instability model. The surface density of solids in disks should scale with the total disk mass [148]. Therefore, if disk mass is correlated with stellar mass, and giant planets form via core accretion, then there is an expected trend for more giant planets around higher mass stars $[149,150]$. The frequency of giant planets arising from disk instability is thought to have no relationship with stellar mass [151].

It should again be emphasized that the giant planet-stellar mass correlation is still in its preliminary form. Surveys at both stellar mass ends are continuing and their results will help bring the true underlying relationship into focus. In the future, it will be interesting to take the next step in this area by investigating how the frequency of solid planets correlates with stellar mass. 


\section{Conclusion}

We have condensed the main results derived from the study of more than 250 exoplanetary systems over 15 years into a coherent picture that can be used to constrain theories of planet formation and evolution. This amalgamation presumes that a coherent picture exists in the first place, and by necessity washes out the individual characteristics of the systems considered. As discussed extensively above, current observational capabilities are still limited, so the field of observational studies of exoplanets is still progressing and often at a rapid pace. Looking toward the future, the two big issues to tackle observationally as technology improves and observational time baselines are extended are determining the orbital, physical, and host star properties of very low mass exoplanets; and the nature of the exoplanets orbiting beyond $3 \mathrm{AU}$. However, despite current limitations, the existing data have already proved enlightening. A more in-depth interpretation of the results as they pertain to the various mechanisms and processes of planet formation and evolution are discussed in the following chapters.

\section{References}

1 Mayor, M. and Queloz, D. (1995) Nature, 378, 355.

2 Rivera, E.J., Lissauer, J.J., Butler, R.P. et al. (2005) The Astrophysical Journal, 634, 625.

3 Benedict, G.F., McArthur, B.E., Forveille, T. et al. (2002) The Astrophysical Journal, 581, 115.

4 Zucker, S. and Mazeh, T. (2001) The Astrophysical Journal, 562, 1038.

5 Jorissen, A., Mayor, M., and Udry, S. (2001) Astronomy and Astrophysics, 379, 992.

6 Bean, J.L., McArthur, B.E., Benedict, G.F. et al. (2007) The Astronomical Journal, 134, 749.

7 Ford, E.B. (2005) The Astronomical Journal, 129, 1706.

8 Saar, S.H., Butler, R.P., and Marcy, G.W. (1998) The Astrophysical Journal, 498, 153.

9 Wright, J.T. (2005) The Publications of the Astronomical Society of the Pacific, 117, 657.

10 Desort, M., Lagrange, A.-M., Galland, F. et al. (2007) Astronomy and Astrophysics, 473, 983.
11 Queloz, D., Henry, G.W., Sivan, J.P. et al. (2001) Astronomy and Astrophysics, 379, 279.

12 Hatzes, A.P., Cochran, W.D., McArthur, B. et al. (2000) The Astrophysical Journal, 544, 145.

13 Paulson, D.B., Cochran, W.D., and Hatzes, A.P. (2004) The Astronomical Journal, 127, 3579.

14 Paulson, D.B. and Yelda, S. (2006) The Publications of the Astronomical Society of the Pacific, 118, 706.

15 Udry, S., Mayor, M., Naef, D. et al. (2000) Astronomy and Astrophysics, 356, 590.

16 Marcy, G., Butler, R.P., Fischer, D. et al. (2005) Progress of Theoretical Physics Supplement, 158, 24.

17 Johnson, J.A., Butler, R.P., Marcy, G.W. et al. (2007) The Astrophysical Journal, 670, 833.

18 Mayor, M., Udry, S., Lovis, C. et al. (2009) Astronomy and Astrophysics, 493, 639.

19 Forveille, T., Bonfils, X., Delfosse, X. et al. (2009) Astronomy and Astrophysics, 493, 649. 
22 1 Exoplanet Observations

20 Hebb, L., Collier-Cameron, A., Loeillet, B. et al. (2009) The Astrophysical Journal, 693, 1920.

21 Mazeh, T., Zucker, S., and Pont, F. (2005) Monthly Notices of the Royal Astronomical Society, 356, 955.

22 Torres, G., Winn, J.N., and Holman, M.J. (2008) The Astrophysical Journal, 677, 1324.

23 Wright, J.T., Marcy, G.W., Butler, R.P. et al. (2008) The Astrophysical Journal, 683, 63.

24 Wright, J.T., Marcy, G.W., Fischer, D.A. et al. (2007) The Astrophysical Journal, 657, 533.

25 Kalas, P., Graham, J.R., Chiang, E. et al. (2008) Science, 322, 1345.

26 Marois, C., Macintosh, B., Barman, T. et al. (2008) Science, 322, 1348

27 Lagrange, A.-M., Gratadour, D., Chauvin, G. et al. (2009) Astronomy and Astrophysics, 493, 21.

28 Lafreniére, D., Doyon, R., Marois, C. et al. (2007) The Astrophysical Journal, 670, 1367.

29 Nielsen, E.L., Close, L.M., Biller, B.A. et al. (2008) The Astrophysical Journal, 674, 466.

30 Laughlin, G., Marcy, G.W., Vogt, S.S. et al. (2005) The Astrophysical Journal, 629, 121.

31 Halbwachs, J.L., Mayor, M., and Udry, S. (2005) Astronomy and Astrophysics, 431, 1129.

32 Laughlin, G. and Adams, F.C. (1999) The Astrophysical Journal, 526, 88.

33 Barnes, R. and Quinn, T. (2004) The Astrophysical Journal, 611, 494.

34 Correia, A.C.M., Udry, S., Mayor, M. et al. (2005) Astronomy and Astrophysics, 440, 751.

35 Pepe, F., Correia, A.C.M., Mayor, M. et al. (2007) Astronomy and Astrophysics, 462, 769 .

36 Goździewski, K. and Maciejewski, A.J. (2001) The Astrophysical Journal, 563, 81.

37 Laughlin, G. and Chambers, J.E. (2001) The Astrophysical Journal, 551, 109.

38 Rivera, E.J. and Lissauer, J.J. (2001) The Astrophysical Journal, 558, 392.

39 Ribas, I., Font-Ribera, A., and Beaulieu, J.-P. (2008) Astronomy and Astrophysics, 677, 59.
40 Alonso, R., Barbieri, M., Rabus, M., Deeg, H.J. et al. (2008) Astronomy and Astrophysics, 487, 5.

41 Bean, J.L. and Seifahrt, A. (2008) Astronomy and Astrophysics, 487, 25.

42 Bean, J.L. and Seifahrt, A. (2009) Astronomy and Astrophysics, 496, 249.

43 Wright, J.T., Upadhyay, S., Marcy, G.W et al. (2009) The Astrophysical Journal, 693, 1084.

44 Fischer, D.A., Marcy, G.W., Butler, R.P. et al. (2008) The Astrophysical Journal, 675, 790 .

45 Bean, J.L., McArthur, B.E., Benedict, G.F., and Armstrong, A. (2008) The Astrophysical Journal, 672, 1202.

46 Butler, R.P., Wright, J.T., Marcy, G.W. et al. (2006) The Astrophysical Journal, 646, 505.

47 Lee, M.H. (2004) The Astrophysical Journal, 611, 517.

48 Duquennoy, A. and Mayor, M. (1991) Astronomy and Astrophysics, 248, 485.

49 Lada, C.J. (2006) The Astrophysical Journal, 640, 63.

50 Mugrauer, M., Seifahrt, A., and Neuhäuser, R. (2007) Monthly Notices of the Royal Astronomical Society, 378, 1328.

51 Eggenberger, A., Udry, S., Chauvin, G. et al. (2007) Astronomy and Astrophysics, 474, 273.

52 Mugrauer, M., Neuhäuser, R., and Mazeh, T. (2007) Astronomy and Astrophysics, 469, 755.

53 Mugrauer, M., Neuhäuser, R., Seifahrt, A. et al. (2005) Astronomy and Astrophysics, 440, 1051.

54 Zucker, S., Mazeh, T., Santos, N.C. et al. (2003) Astronomy and Astrophysics, 404, 775.

55 Konacki, M. (2005) The Astrophysical Journal, 626, 431.

56 Konacki, M. (2005) Nature, 436, 230.

57 Eggenberger, A., Udry, S., Mazeh, T. et al. (2007) Astronomy and Astrophysics, 466, 1179.

58 Queloz, D., Mayor, M., Weber, L. et al. (2000) Astronomy and Astrophysics, 354, 99.

59 Hatzes, A.P., Cochran, W.D., Endl, M. et al. (2003) The Astrophysical Journal, 599, 1383. 
60 Zucker, S., Mazeh, T., Santos, N.C. et al. (2004) Astronomy and Astrophysics, 426, 695.

61 Pichardo, B., Sparke, L.S., and Aguilar, L.A. (2005) Monthly Notices of the Royal Astronomical Society, 359, 521.

62 Jang-Condell, H., Mugrauer, M., and Schmidt, T. (2008) The Astrophysical Journal, 683, 191

63 Zucker, S. and Mazeh, T. (2002) The Astrophysical Journal, 568, 113.

64 Eggenberger, A., Udry, S., and Mayor, M. (2004) Astronomy and Astrophysics, 417, 353.

65 Rossiter, R.A. (1924) The Astrophysical Journal, 60, 15.

66 McLaughlin, D.B. (1924) The Astrophysical Journal, 60, 22.

67 Gaudi, B.S. and Winn, J.N. (2007) The Astrophysical Journal, 655, 550.

68 Winn, J.N., Noyes, R.W., Holman, M.J. et al. (2005) The Astrophysical Journal, 631, 1215.

69 Winn, J.N., Johnson, J.A., Marcy, G.W. et al. (2006) The Astrophysical Journal, 653, 69.

70 Winn, J.N., Johnson, J.A., Peek, K.M.G. et al. (2007) The Astrophysical Journal, 665, 167.

71 Loeillet, B., Shporer, A., Bouchy, F. et al. (2008) Astronomy and Astrophysics, 481, 529.

72 Narita, N., Enya, K., Sato, B. et al. (2007) The Publications of the Astronomical Society of Japan, 59, 763.

73 Wolf, A.S., Laughlin, G., Henry, G.W. et al. (2007) The Astrophysical Journal, 667, 549.

74 Bouchy, F., Queloz, D., Deleuil, M. et al. (2008) Astronomy and Astrophysics, 482, 25.

75 Narita, N., Sato, B., Ohshima, O., and Winn, J.N. (2008) The Publications of the Astronomical Society of Japan, 60, 1.

76 Cochran, W.D., Redfield, S., Endl, M., and Cochran, A.L. (2008) The Astrophysical Journal, 683, 59.

77 Hébrard, G., Bouchy, F., Pont, F. et al. (2008) Astronomy and Astrophysics, 488, 763.

78 Winn, J.N., Johnson, J.A., Fabrycky, D. et al. The Astrophysical Journal, 700, 302, arXiv:0902.3461.
79 Winn, J.N., Johnson, J.A., Narita, N. et al. (2008) The Astrophysical Journal, 682, 1283.

80 Johnson, J.A., Winn, J.N., Narita, N. et al. (2008) The Astrophysical Journal, 686, 649 .

81 Cumming, A., Butler, R.P., Marcy, G.W. et al. (2008) The Publications of the Astronomical Society of the Pacific, 120, 531.

82 Bean, J.L., Benedict, G.F., Charbonneau, D. et al. (2008) Astronomy and Astrophysics, 486, 1039.

83 Sousa, S.G., Santos, N.C., Mayor, M. et al. (2008) Astronomy and Astrophysics, 487, 373.

84 Beaulieu, J.-P., Bennett, D.P., Fouqué, P. et al. (2006) Nature, 439, 437

85 Bennett, D.P., Bond, I.A., Udalski, A et al. (2008) The Astrophysical Journal, 684, 663.

86 Winn, J.N., Holman, M.J., Torres, G. et al. (2008) The Astrophysical Journal, 683, 1076.

87 Irwin, J., Charbonneau, D., Nutzman, P. et al. (2008) The Astrophysical Journal, 681, 636.

88 Pál, A., Bakos, G.A., Torres, G. et al. (2008) The Astrophysical Journal, 680 , 1450.

89 Pont, F., Tamuz, O., Udalski, A. et al. (2008) Astronomy and Astrophysics, 487, 749 .

90 Udalski, A., Pont, F., Naef, D. et al. (2008) Astronomy and Astrophysics, 482, 299.

91 Barge, P., Baglin, A., Auvergne, M. et al. (2008) Astronomy and Astrophysics, 482, 17.

92 Alonso, R., Auvergne, M., Baglin, A. et al. (2008) Astronomy and Astrophysics, 482, 21.

93 Moutou, C., Bruntt, H., Guillot, T. et al. (2008) Astronomy and Astrophysics, 488, 47.

94 Anderson, D.R., Gillon, M., Hellier, C. et al. (2008) Monthly Notices of the Royal Astronomical Society, 387, 4.

95 Wilson, D.M., Gillon, M., Hellier, C. et al. (2008) The Astrophysical Journal, 675, 113.

96 Pollacco, D., Skillen, I., Collier Cameron, A. et al. (2008) 
Monthly Notices of the Royal Astronomical Society, 385, 1576.

97 Burke, C.J., McCullough, P.R., Valenti, J.A. et al. (2008) The Astrophysical Journal, 686, 1331.

98 Shporer, A., Bakos, G.A., Bouchy, F. et al. (2009) The Astrophysical Journal, 690, 1393.

99 Hellier, C., Anderson, D.R., Gillon, M. et al. (2009) The Astrophysical Journal, $690,89$.

100 West, R.G., Anderson, D.R., Gillon, M. et al. (2009) The Astronomical Journal, 137, 483.

101 Joshi, Y.C., Pollacco, D., Cameron, A.C et al. (2009) Monthly Notices of the Royal Astronomical Society, 392, 1532.

102 Bakos, G.A., Pál, A., Torres, G. et al. (2009) The Astrophysical Journal, 696, 1950

103 Johnson, J.A., Winn, J.N., Cabrera, N.E., and Carter, J.A. (2009) The Astrophysical Journal, 692, 100.

104 Fortney, J.J., Marley, M.S., and Barnes, J.W. (2007) The Astrophysical Journal, 659, 1661.

105 Charbonneau, D., Brown, T.M., Latham, D.W., and Mayor, M. (2000) The Astrophysical Journal, 529, 45

106 Fabrycky, D.C., Johnson, E.T., and Goodman, J. (2007) The Astrophysical Journal, 665, 754.

107 Bodenheimer, P., Laughlin, G., and Lin, D.N.C. (2003) The Astrophysical Journal, 592, 555.

108 Jackson, B., Greenberg, R., and Barnes, R. (2008) The Astrophysical Journal, 681, 1631

109 Sato, B., Fischer, D.A., Henry, G.W. et al. (2005) The Astrophysical Journal, 633, 465.

110 Carter, J.A., Winn, J.N., Gilliland, R., and Holman, M.J. (2009) The Astrophysical Journal, 696, 241.

111 Ikoma, M., Guillot, T., Genda, H. et al. (2006) Astronomy and Astrophysics, 650, 1150.

112 Baraffe, I., Chabrier, G., and Barman, T. (2008) Astronomy and Astrophysics, $482,315$.

113 Dodson-Robinson, S.E. and Bodenheimer, P. (2009) The Astrophysical Journal, 695, 159.
114 Gonzalez, G. (1997) Monthly Notices of the Royal Astronomical Society, 285, 403.

115 Santos, N.C., Israelian, G., Mayor, M. et al. (2005) Astronomy and Astrophysics, 437, 1127

116 Fischer, D.A. and Valenti, J. (2005) The Astrophysical Journal, 622, 1102

117 Bodaghee, A., Santos, N.C., Israelian, G., and Mayor, M. (2003) Astronomy and Astrophysics, 404, 715.

118 Gilli, G., Israelian, G., Ecuvillon, A. et al. (2006) Astronomy and Astrophysics, 449, 723.

119 Laws, C., Gonzalez, G., Walker, K.M. et al. (2003) The Astronomical Journal, 125, 2664

120 Bond, J.C., Tinney, C.G., Butler, R.P. et al. (2006) Monthly Notices of the Royal Astronomical Society, 370, 163.

121 Santos, N.C., Israelian, G., Mayor, M. et al. (2003) Astronomy and Astrophysics, 398, 363.

122 Ecuvillon, A., Israelian, G., Santos, N.C. et al. (2006) Astronomy and Astrophysics, 449, 809.

123 Paulson, D.B., Sneden, C., and Cochran, W.D. (2003) The Astronomical Journal, 125, 3185.

124 Desidera, S., Gratton, R.G., Scuderi, S. et al. (2004) Astronomy and Astrophysics, 420, 683.

125 Desidera, S., Gratton, R.G., Lucatello, S et al. (2006) Astronomy and Astrophysics, 454, 581.

126 Reddy, B.E., Lambert, D.L., Laws, C. et al. (2002) Monthly Notices of the Royal Astronomical Society, 335, 1005.

127 Vauclair, S., Laymand, M., Bouchy, F. et al. (2008) Astronomy and Astrophysics, 482, 5.

128 Santos, N.C., Israelian, G., and Mayor, M. (2004) Astronomy and Astrophysics, 415, 1153

129 Pollack, J.B., Hubickyj, O., Bodenheimer, P. et al. (1996) Icarus, 124, 62.

130 Mayer, L., Lufkin, G., Quinn, T., and Wadsley, J. (2007) The Astrophysical Journal, 661, 77.

131 Boss, A. (2002) The Astrophysical Journal, 567, 149.

132 Matsuo, T., Shibai, H., Ootsubo, T., and Tamura, M. (2007) The Astrophysical Journal, 662, 1282. 
133 Fuhrmann, K. (1998) Astronomy and Astrophysics, 338, 161.

134 da Silva, L., Girardi, L., Pasquini, L. et al. (2006) Astronomy and Astrophysics, 458, 609.

135 Cochran, W.D., Endl, M., Wittenmyer, R., and Bean, J.L. (2007) The Astrophysical Journal, 665, 1407.

136 Sozzetti, A., Torres, G., Latham, D.W. et al. (2006) The Astrophysical Journal, 649, 428.

137 Guillot, T., Santos, N.C., Pont, F. et al. (2006) Astronomy and Astrophysics, 453 21.

138 Burrows, A., Hubeny, I., Budaj, J., and Hubbard, W.B. (2007) The Astrophysical Journal, 661, 502

139 Robinson, S.E., Laughlin, G. Bodenheimer, P., and Fischer, D. (2006) The Astrophysical Journal, 643, 484

140 Butler, R.P., Johnson, J.A., Marcy, G.W. et al. (2006) The Publications of the Astro nomical Society of the Pacific, 118, 1685.

141 Endl, M., Cochran, W.D., Kürster, M. et al. (2006) The Astrophysical Journal, 649, 436.

142 Bonfils, X., Mayor, M., Delfosse, X. et al. (2007) Astronomy and Astrophysics, 474, 293.

143 Sato, B., Ando, H., Kambe, E. et al. (2003) The Astrophysical Journal, 597, 157.
144 Setiawan, J., Pasquini, L., da Silva, L. et al. (2004) Astronomy and Astrophysics, 421, 241.

145 Lovis, C. and Mayor, M. (2007) Astronomy and Astrophysics, 472, 657.

146 Cayrel de Strobel, G., Soubiran, C., and Ralite, N. (2001) Astronomy and Astrophysics, 373, 159.

147 Bean, J.L., Sneden, C., Hauschildt, P.H. et al. (2006) The Astrophysical Journal, 652, 1604.

148 Ida, S. and Lin, D.N.C. (2005) The Astrophysical Journal, 626, 1045.

149 Laughlin, G., Bodenheimer, P., and Adams, F.C. (2004) The Astrophysical Journal, 612, 73.

150 Kennedy, G.M. and Kenyon, S.J. (2008) The Astrophysical Journal, 673, 502.

151 Boss, A. (2006) The Astrophysical Journal, 643, 501.

\section{Further Reading}

Johns-Krull, C.M., McCullough, P.R., Burke, C.J. et al. (2008) The Astrophysical Journal, 677, 657. 
